Mathematics in Applied Sciences and Engineering

Volume 2, Number 1, March 2021, pp.10-21

https://ojs.lib.uwo.ca/mase

https://doi.org/10.5206/mase/10093

\title{
A LEADER-FOLLOWERS GAME OF EMERGENCY PREPAREDNESS FOR ADVERSE EVENTS
}

\author{
MYLES NAHIRNIAK, MONICA COJOCARU, AND TANGI MIGOT
}

\begin{abstract}
Natural disasters occur across the globe, resulting in billions of dollars of damage each year. Effective preparation before a disaster can help to minimize damages, economic impact, and loss of human life. This paper uses a game theory framework to set up a leader-followers model for resource distribution to several geographic zones before an adverse event. The researchers model population members who may choose to prepare in advance of an event by acquiring supplies, whereas others may wait until the last minute. Failure to prepare in advance could result in a significant loss due to the chance that supplies may no longer be available. Numerical simulations are run to determine how the leader should distribute supplies to maximize the preparedness of the overall population. It was found that population size is a significant factor for supply distribution, but the behaviour of individuals within a zone is also important. Much of the current resource allocation research focuses on the logistics and economics of supply distribution, but this paper demonstrates that social aspects should also be considered.
\end{abstract}

\section{INTRODUCTION}

Throughout 2019 in the United States alone, there were 14 natural disasters each causing 1 billion USD or more of damage [13]. One example of such a disaster is hurricane Dorian, which in August and September affected several Caribbean nations, most notably the Bahamas, as well as the Eastern United States and provinces in Atlantic Canada. Effective preparation in advance of a disaster is necessary to mitigate damages, financial costs, and loss of human life.

A review by Altay and Green [1] identified a lack of cooperation between humanitarian agencies. As a response, Galindo and Batta [5] recommended modelling a leader agency that is responsible for overall coordination. Muggy and Heier Stamm [10] agreed that cooperative models are lacking, and determined that game theory is an appropriate tool for determining an optimal allocation of resources.

Disaster relief is divided into four stages, known as mitigation, preparedness, response, and recovery 8. Our motivation for this paper is to use a bilevel game to model the distribution of supplies among various geographical zones in preparation for a disaster, while also considering how members of the population choose to prepare. Many existing environmental and disaster relief models which use a game theory framework consider cases where relief agencies or suppliers either compete or cooperate. It is known that two parties cooperating to implement an environmental project is more effective than each party working independently $[3]$.

Previous studies have examined socio-cognitive reasons that members of a population may or may not prepare for adverse events, however these factors have not been included in mathematical models. Even with advance knowledge, many individuals will choose not to prepare for a natural disaster [15]. The level of preparation amongst a population can depend on the ease of acquiring supplies, as well their ease of implementation [14. Another issue is the public's compliance with recommended safety

Received by the editors 20 October 2020; accepted 12 January 201; published online 22 January 2021.

2000 Mathematics Subject Classification. 91A40, 91B32, 91A65. 
measures put forward by authorites; some population members may not have trust in the authority while others believe the adverse event will be less severe than predicted [2]. Finally, Lopes [7] determined that individuals overestimate their level of preparedness compared to their actual level of preparedness. In our paper, we posit that social aspects are significant, and incorporate these factors in our model.

There is much literature with the focus on optimizing the cost and transport of supplies. Nagurney and Flores [12] develop an equilibrium model to ensure consistent flow of supplies under cost constraints. Similar research was conducted to specifically analyze and reduce the transportation costs of supplies to different sites [6]. These models, however, ignore the problem of how to effectively allocate resources to multiple players. Nagurney et al. [11] adapted a generalized Nash game model for post-disaster relief, focusing on the distribution of funds. Their model assumes multiple organizations that have all decided in advance the amount of relief to be provided at each point.

In contrast, we propose an alternative approach. In our model, we consider how resources should be allocated to multiple geographic zones in advance of a disaster, to ensure that the maximum overall population is prepared. Additionally, we consider the human behaviour aspect of how a population prepares in advance of a disaster. We model the situation as a leader-followers game in which an overseeing party is responsible for distributing supplies among several geographical zones. In each zone, the population is divided into two groups: one who chooses to acquire supplies in advance of a disaster and the other who waits until the last minute, knowing there is a risk that supplies will run out. We consider two other important factors in our analysis: first, we differentiate the populations in each zones not only by their size (total number of inhabitants per zone), but also by their cost of acquiring preparedness supplies in advance. The cost here should not be regarded as a dollar amount, but rather as a personal cost, in time, money and effort to acquire appropriate supplies. Second, we differentiate the zones by assuming different probabilities of running out of distributed supplies and that costs of acquiring supplies may be reduced (by incentives) to nudge individuals to consider supplies in advance, rather than last minute. Reductions in personal costs would be for instance: convenience of locations for supplies distribution centers, distribution of supplies "at residence" for people of higher risk (such as senior citizens or long-term care homes), information campaigns, and "drive-through" or delivery options, etc.

This paper provides an overview of the model, and sets up the problems for the followers and the leader. We provide two types of analyses, based on parameter sensitivity analysis techniques. To begin the analysis in Section 3, we first establish a "base case" scenario of the followers problem, then we complete a sensitivity analysis on their possible best strategies. We continue in Section 4 with a further sensitivity analysis of the leader's best decision based on models parameters and leader's constraints. In section 5 we link the probability of running out of supplies in each zone to the choices of individuals in that zone. We re-evaluate the model under this new assumption, and contrast the results with our previous analyses. We conclude that population density and advance incentives are the most important factors for a leader to consider when allocating supplies.

\section{Game theoretic Resource allocation model in Diverse populations}

In this section, we develop a model for the distribution of resources among several geographical zones. We formulate the model as a leader-followers game. Each zone's followers is described by a symmetric two-player bimatrix game. The leader's problem is formulated as an optimization problem, given the behaviour of the players in each zone. We will explain the parameters and assumptions used in the model below.

Consider a leader to be an authority designated to distribute supplies among $N$ zones, labelled as $n=1, \ldots, N$. This could represent the government, or a charitable organization providing disaster 
relief. For some total quantity of supplies, $Q_{t o t}$, denote the amount distributed to each of the zones as $Q_{n}$, and any leftover supplies not distributed to be $Q_{\text {remain }}$.

The total supplies are $Q_{t o t}=\sum_{n=1}^{N} Q_{n}+Q_{\text {remain }}$. The fraction of total supplies provided to a given zone is $w_{n}=\frac{Q_{n}}{Q_{t o t}}$ which implies $\sum_{n=1}^{N} w_{n} \leq 1$.

Represent the population of each zone by $P_{n}$. The population spread among the zones is given by $p_{n}=\frac{P_{n}}{\sum_{n=1}^{N} P_{n}}$, where $p_{n}$ is the fraction of the total population in zone $n$.

2.1. Followers' game. We now assume that the players in each of the zones play the same game. Their two pure strategies are represented by: 1 = "stocking up on supplies before an adverse event", or 2 = "waiting until the last minute to purchase supplies". Advance purchasing may be viewed as carrying a lower personal cost to a player, whereas last minute search has a risk that supplies may have run out and the player may not be able to acquire needed supplies. We model the players' choices as a symmetric bimatrix game in each zone, where the two players share the same matrix, $A$, of payoffs given by

$$
\begin{array}{cc}
y_{n} & 1-y_{n} \\
x_{n} & \left(\begin{array}{cc}
T_{n}-C_{n} & T_{n}-\gamma C_{n} \\
T_{n}-\gamma C_{n} & T_{n}-\left(q_{n} L_{n}+\left(1-q_{n}\right) 2 C_{n}\right)
\end{array}\right) .
\end{array}
$$

In the above bimatrix, $x_{n}$ represents the frequency that player 1 in zone $n$ plays strategy $1=$ "supply in advance". Because each player may only choose between one of two strategies, the value $1-x_{n}$ is the frequency that player 1 in zone $n$ plays strategy $2=$ "supply last minute". Similarly, $y_{n}$ and $1-y_{n}$ are the frequencies that player 2 in zone $n$ plays strategies 1 and 2, respectively.

The "budget" available to each player in zone $n$ is denoted $T_{n}$, and the cost of acquiring supplies is $C_{n}$. There is a probability of running out of supplies, $q_{n}$, which leads to the potential for either a large loss, $L_{n}$, or for acquiring supplies at a larger personal cost. We assume that at the last minute, the remaining supplies require double the effort/cost to be acquired. There is an incentive factor for the cost of supplies if individuals from a single zone supply in advance, denoted by $\gamma$. With no incentive $(\gamma=0)$, individuals pay full price; a high value of $\gamma$ provides an incentive to supply in advance, thus lowering the effective cost of supplies. This can signify, for example, that the time they spent for acquiring supplies is very short, or that supply distribution centres were conveniently located and well-stocked.

The parameters in the model, $T_{n}, C_{n}, L_{n}, q_{n}$, and $\gamma$ are normalized to be between 0 and 1 . We assume further that a player must have enough funds to purchase supplies if desired, and the potential loss is greater than the cost of supplies, so $0 \leq C_{n}<L_{n} \leq T_{n}$. The expected payoff $E_{n}$ for a player in zone $n$ is given by

$$
E_{n}\left(x_{n}, y_{n}\right)=\left(x_{n}, 1-x_{n}\right) A\left(y_{n}, 1-y_{n}\right)^{T} .
$$

A strategy $\left(x_{n}^{*}, y_{n}^{*}\right)$ in zone $n$ is a Nash equilibrium if $x_{n}^{*}$ is the best response to $y_{n}^{*}$ and vice versa, that is

$$
E_{n}\left(x_{n}^{*}, y_{n}^{*}\right) \geq E_{n}\left(x_{n}^{*}, y_{n}\right), \forall y_{n} \in[0,1]
$$

and since the game is symmetric, then $x_{n}^{*}=y_{n}^{*}$. Direct computation allows us to determine the equilibrium points for the game. We find that the mixed equilibrium in the zone $n$ is given by:

$$
x_{n}^{*}=\frac{C_{n} \gamma+2 C_{n} q_{n}-2 C_{n}-L_{n} q_{n}}{2 C_{n} \gamma+2 C_{n} q_{n}-3 C_{n}-2 q_{n}} .
$$

We verify that $0 \leq x_{n}^{*} \leq 1$ in Section 3

2.2. Leader's problem. The leader's goal is to supply as much of the population as possible, in advance of an event. This is done by optimizing the allocation of supplies to each zone and choosing appropriate incentives in the distribution process that would amount to a lesser personal $\operatorname{cost} C_{n}$ for a 
player to supply in advance. We assume that the probability of running out of supplies $q_{n}$ is different for each zone, depending on the behaviour of the population in that zone and the amount of supplies, $w_{n}$, distributed.

The leader's problem then is given by:

$$
\begin{array}{ll}
\max _{(w, \gamma, x)} \theta_{L}:=\sum_{n=1}^{N} p_{n} x_{n} \\
\text { s.t. } \quad\left(w_{1}, \ldots, w_{N}, \gamma\right) \in[0,1]^{N+1}, \\
0 \leq \sum_{n=1}^{N} w_{n} \leq 1, \\
\quad x_{n} \in \arg \max _{x_{n}}\left\{E_{n}: 0 \leq x_{n} \leq 1\right\}, \forall n .
\end{array}
$$

\section{Sensitivity analysis of followers' optimal StRategies in a 2-ZONE ALLOCATion PROBLEM WITH FIXED COSTS AND LOSSES FOR FOLLOWERS}

For ease of presentation, let us consider the leader's problem (2.1), with $N=2$ (i.e. for two zones). The leader's function for two zones now becomes:

$$
\theta_{L}\left(w_{1}, w_{2}, \gamma, x^{*}\right)=p_{1} x_{1}^{*}\left(p_{1}, w_{1}\right)+\left(1-p_{1}\right) x_{2}^{*}\left(p_{2}, w_{2}\right) .
$$

using the solution points from the followers' games:

$$
\begin{aligned}
& x_{1}^{*}=\frac{C_{1} \gamma+2 C_{1} q_{1}-2 C_{1}-L_{1} q_{1}}{2 C_{1} \gamma+2 C_{1} q_{1}-3 C_{1}-2 q_{1}} \\
& x_{2}^{*}=\frac{C_{2} \gamma+2 C_{2} q_{2}-2 C_{2}-L_{2} q_{2}}{2 C_{2} \gamma+2 C_{2} q_{2}-3 C_{2}-2 q_{2}} .
\end{aligned}
$$

To analyze the leader's problem, we assume here an equal population distribution between the two zones $\left(p_{1}=p_{2}=0.5\right)$ and an equitable distribution of resources of $w_{1}=w_{1}=0.5$, then the leader's function becomes $\theta_{L}=0.5 x_{1}^{*}+0.5 x_{2}^{*}$. The leader's function is maximized when the followers' best strategy is maximal. Thus we will strive to analyze the optimal strategy values for the followers.

3.1. Base case. To better understand the problem, we now define a base case scenario, that is: $L_{1}=L_{2}$ and $C_{1}=C_{2}$, with $C_{1}<L_{1}$. We then first compute the followers' best strategy $x_{1}^{*}$ depending on possible values of $\gamma, q_{1}$.

We draw the evolution of $x_{1}^{*}$ as a function of $\gamma$ and $q_{1}$ in the base case where $C_{1}=C_{2}=0.5$ and $L_{1}=L_{2}=0.75$ in Figure 1. We see that, indeed, the values of the follower's best strategy lie in $[0,1]$. Assuming an equal population distribution between the two zones $\left(p_{1}=p_{2}=0.5\right)$ and an equitable distribution of resources of $w_{1}=w_{1}=0.5$, then the leader's function $\theta_{L}$ is maximized when the followers best strategy is $x_{1}^{*}=x_{2}^{*}=1$ given in Figure 1. We can exactly compute it to be $\theta_{L}=\sum_{n=1}^{2} 0.5 x_{n}^{*}$ with a maximal value of $\theta_{L}=1$ when $\gamma=1, q_{n}=0, n=\{1,2\}$ (i.e. all will supply in advance if incentive $\gamma$ becomes maximal $(\gamma=1)$ and there is no possibility of running out of supplies $\left(q_{1}=q_{2}=0\right)$.

3.2. Deviating from the base case. In this subsection we start to differentiate between the two zones, in the way we setup the follower's input parameters $C_{n}, L_{n}, n=\{1,2\}$. We look at the following two scenarios:

- $C_{2}=1.5 C_{1}$ and $L_{1}=L_{2}=0.75$, where cost in zone 2 is 1.5 times higher than in zone 1 , but losses are comparable;

- $C_{2}=C_{1}$ and $L_{2}=L_{1}+0.15$, where losses in zone 2 are $20 \%$ higher than in zone 1 , but costs are comparable. 


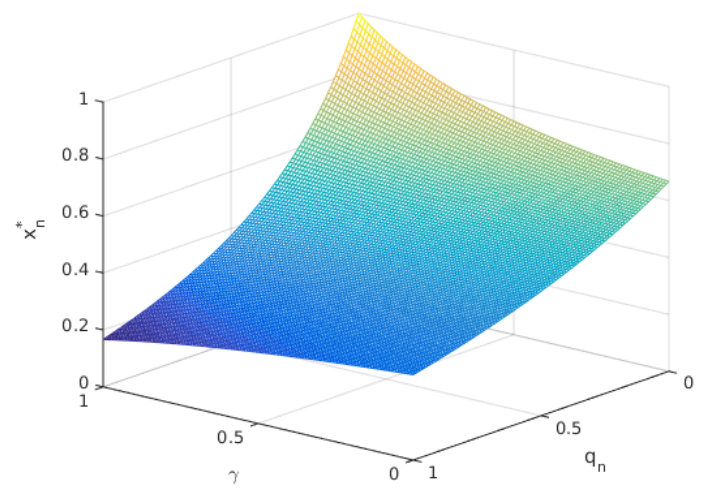

Figure 1. Evolution of $x_{n}^{*}$ as a function of $\gamma$ and $q_{n}$ in the base case where $C_{n}=0.5$ and $L_{n}=0.75, n=\{1,2\}$.

In these cases, the plots of the followers' best strategies are given in Figure 2 and 3 as: We see that

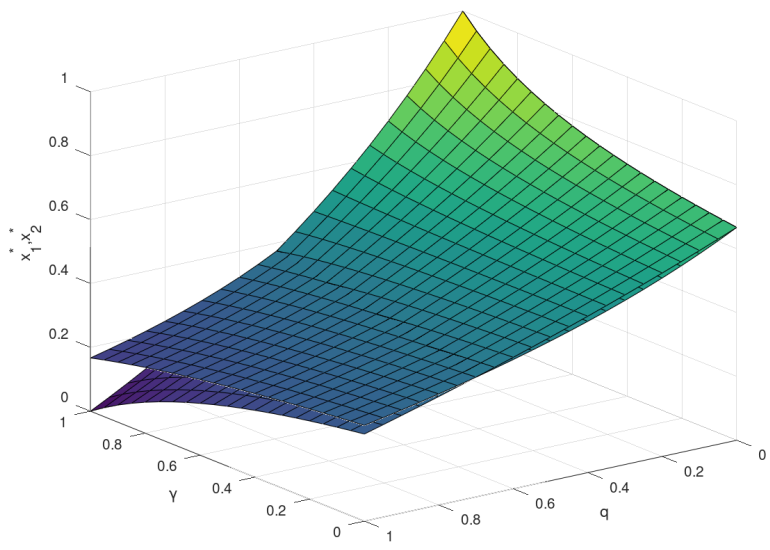

Figure 2. Evolution of $x_{n}^{*}$ as a function of $\gamma$ and $q_{n}$ where $C_{2}=1.5 C_{1}$ and $L_{1}=L_{2}=$ $0.75, n=\{1,2\}$.

the leaders' function, under the base case scenario conditions $\left(p_{1}=p_{2}=0.5\right.$ and $\left.w_{1}=w_{2}=0.5\right)$, is maximized whenever $x_{n}^{*}$ values are maximal for both populations. In both Figure 2 and Figure 3 we see that the leader's function is maximized in the best of circumstances, i.e., all will supply in advance if incentive $\gamma$ becomes maximal $(\gamma=1)$ and there is no possibility of running out of supplies $\left(q_{1}=q_{2}=0\right)$. What is interesting to look at is the least favourable scenario, i.e.: the case where there are no incentives $(\gamma=0)$ and there is a certainty of running out of supplies $\left(q_{1}=q_{2}=1\right)$. Here, when losses are the same between zones (Figure 2), individuals in the zone with higher supply cost will not supply in advance at all; on the other hand, when costs are the same but losses differ (Figure 3 ) then both groups will supply in advance in some measure. This will lead to higher values for the leader's function in the latter case. 


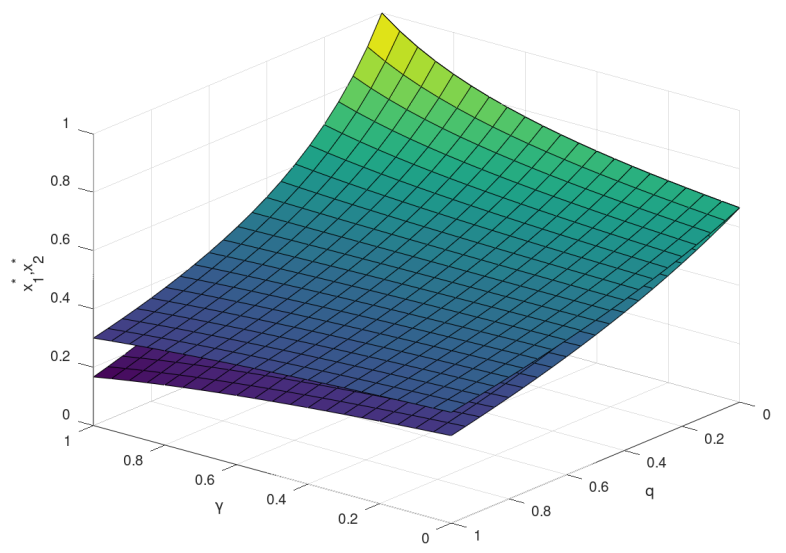

Figure 3. Evolution of $x_{n}^{*}$ as a function of $\gamma$ and $q_{n}$ where $C_{1}=C_{2}=0.5$ and $L_{2}=L_{1}+0.15, n=\{1,2\}$.

\section{Sensitivity analysis of Leader's FunCtion in a 2-ZONe ALlocation PROBlem With fiXed COSTS AND LOSSES FOR FOLLOWERS}

We are now interested to examine a case where costs, as well as losses, are differentiated between zones, but are fixed throughout the analyses. We then present a full sensitivity analysis of the followers' game in Table 1, where the varying parameters are presented in Table 2.

\begin{tabular}{|l|l|l|c|}
\hline$C_{1}$ & 0.5 & $C_{2}$ & 0.75 \\
\hline$L_{1}$ & 0.75 & $L_{2}$ & 0.9 \\
\hline
\end{tabular}

TABLE 1. Table listing the fixed values of input parameters used in this case

\begin{tabular}{|c|l|c|}
\hline$\gamma$ & Discounting factor & {$[0,1]$} \\
\hline$q_{n}$ & Prob. supplies run out in zone $n$ & {$[0,1]$} \\
\hline$w_{n}$ & Supplies fraction to zone $n$ & {$[0,1]$} \\
\hline$p_{1}$ & Population density in zone 1 & {$[0,1]$} \\
\hline$p_{2}$ & Population density in zone 2 & $p_{1}=1-p_{1}$ \\
\hline
\end{tabular}

TABLE 2. Table listing the parameters we vary in the sensitivity analyses below in this problem. Here $n \in\{1,2\}$.

All simulations are conducted by selecting 500 randomly distributed points $\left(\gamma, w_{1}, w_{2}, p_{1}\right) \in[0,1]^{4}$ that satisfy the conditions of the problem, and then computing the corresponding values for the leader's objective function. We note that there is interdependence between the probability of running out of supplies in a zone, $q_{n}$, its population density $p_{n}$, and the allocation of resources $w_{n}$. Specifically, with higher population density in one zone, this probability may increase; on the other hand, with higher allocation of supplies, this probability may decrease.

Let us consider that the probability $q_{n}$ is proportional to $p_{n}$ and inversely proportional to $w_{n}$. To ensure that $0 \leq q_{n} \leq 1$, we choose $q_{1}=\min \left(1, \frac{p_{1}}{\sqrt{w_{1}}}-p_{1}\right)$ and $q_{2}=\min \left(1, \frac{1-p_{1}}{\sqrt{w_{2}}}-\left(1-p_{1}\right)\right)$. We show 
the plot of this functional dependency in Figure 4 below, and note that $q_{n}$ saturates at 1 for large populations if supplies are not sufficiently allocated.

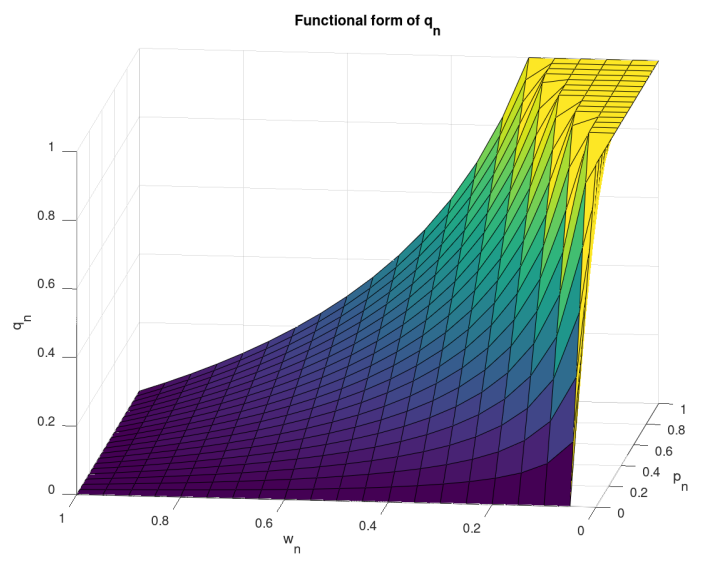

FIGURE 4. Plot of $q_{n}$ as a function of $p_{n}$ and $w_{n}, n=\{1,2\}$.

We use the following subcases to present our results:

(1) We can observe the effect of varying the incentive factor, $\gamma$.

(2) We consider how the population distribution $p_{n}$ between zones affects the allocation weights $w_{1}, w_{2}$.

(3) Finally, we distribute all parameters freely to determine the optimal values of the leader's function.

Case 1.

Using a 30-70\% population split, we show two scenarios: Figure 5 shows the effect of $\gamma$ while freely distributing supplies (left panel), and with all supplies distributed using $w_{1}+w_{2}=1$ (right panel).
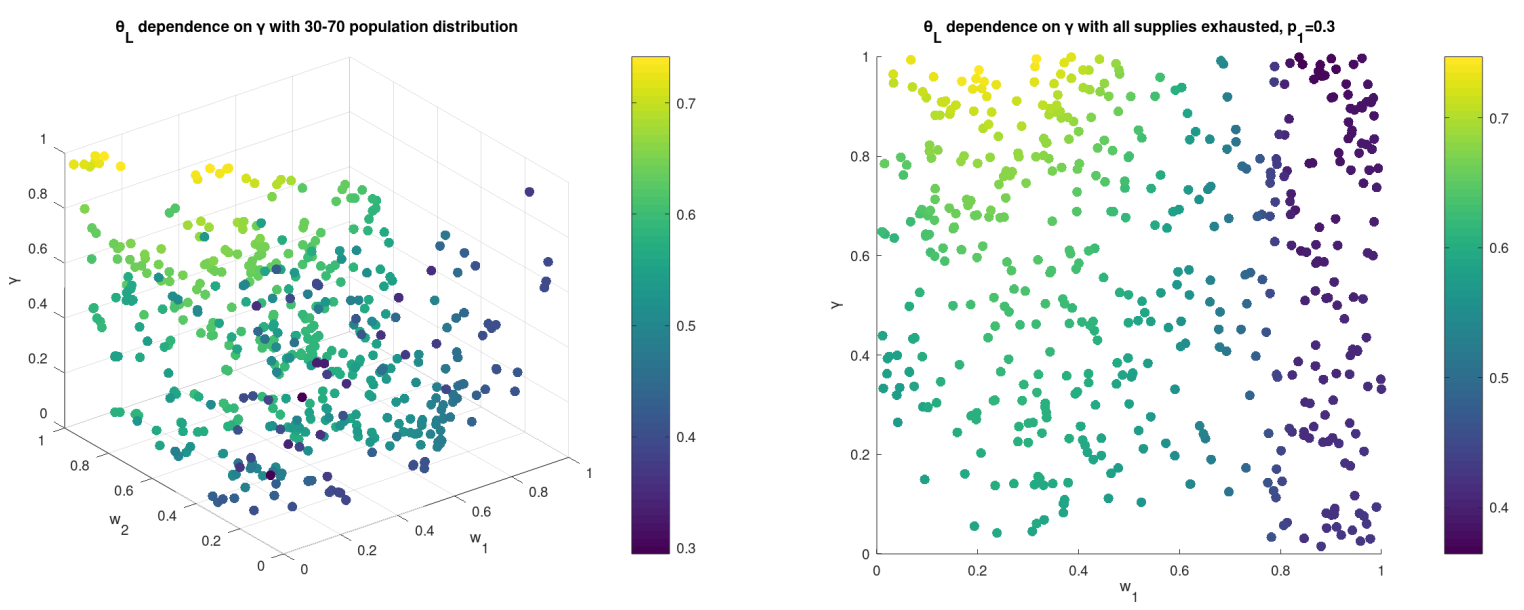

Figure 5. Three parameters are freely distributed: $\left(w_{1}, w_{2} \leq 1-w_{1}, \gamma\right)$ while $p_{1}=0.3$, $q_{1}=\frac{p_{1}}{\sqrt{w_{1}}}-p_{1}$ and $q_{2}=\frac{1-p_{1}}{\sqrt{w_{2}}}-\left(1-p_{1}\right)$. In the right panel we depict the case where supplies are exhausted, i.e. $w_{1}+w_{2}=1$ 
The incentive factor has a strong effect for large $\gamma$, and it can be seen that the objective function is much higher towards the top of the plot in the yellow zone (where $\gamma=1$ ). This indicates that incentivizing the purchase of supplies at a lower effective cost increases the value of the leader's objective function and allows more of the population to prepare in advance. Additionally, below $\gamma=0.6$, there is very little variation in the objective function values. Hence values of $\gamma \geq 0.6$ affect supply distribution.

Case 2. To observe the effect of varying population density between the two zones, we consider two scenarios: Figure 7 shows the effect of $p_{1}$ while freely distributing supplies, as well as distributing all supplies using $w_{1}+w_{2}=1$. The highest objective values occur where the proportion of supplies distributed to a zone is comparable to the fraction of the population in that zone. For the purposes of illustration, a constant value of $\gamma=0.5$ was used in both simulations, but the same conclusions can be drawn for any other value of $\gamma \in[0,1]$.
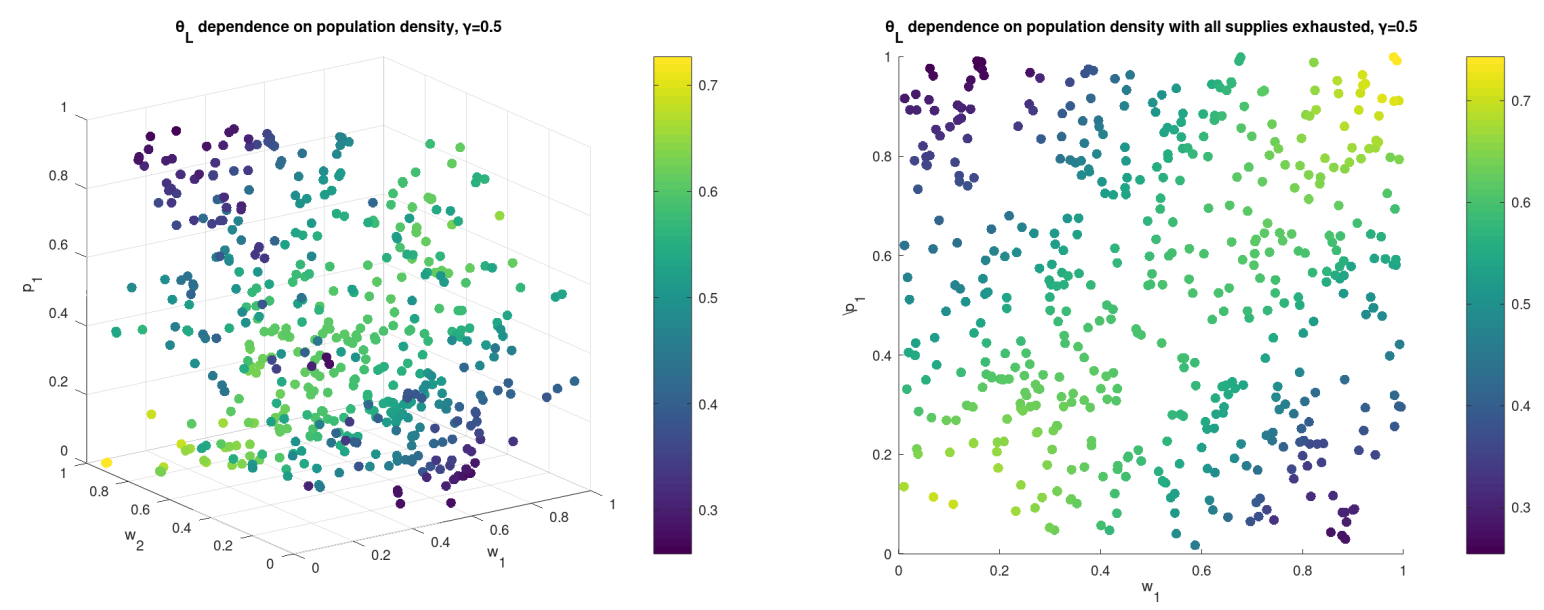

Figure 6. Two parameters are freely distributed: $\left(w_{1}\right.$ and $\left.p_{1}\right)$ while $\gamma=0.5, q_{1}=$ $\frac{p_{1}}{\sqrt{w_{1}}}-p_{1}$ and $q_{2}=\frac{1-p_{1}}{\sqrt{w_{2}}}-\left(1-p_{1}\right)$. In the left panel, $w_{2}$ is freely distributed with the constraint $w_{1}+w_{2} \leq 1$, whereas the right panel depicts the case where supplies are exhausted, with $w_{1}+w_{2}=1$.

Case 3. Here the parameters $\left(w_{1}, w_{2}, p_{1}, \gamma\right)$ are freely distributed, with $p_{2}=1-p_{1}$, and results are shown in Figure 8 . The weights and population size are plotted along the axes, with $\gamma$ being represented by the size of the marker at each point.

The optimal points occur in the top right $\left(p_{1}=1\right.$ and $\left.w_{1}=1\right)$ and lower left $\left(p_{1}=0\right.$ and $\left.w_{1}=0\right)$ regions of Figure $8 \mathrm{a}$. These correspond to the extreme cases where all of the population is in a single zone, and all supplies distributed to that zone, suggesting that higher weights are beneficial to the zone with higher population density. For mixed population distributions, the objective function is optimal when all supplies are distributed, i. e., $w_{1}+w_{2}=1$ We also notice that higher values of $\gamma$ increase the objective function, as large-sized points (high $\gamma$ ) close to small-sized points (low $\gamma$ ) have higher objective values. With all supplies distributed, as shown in Figure 8b, higher $\gamma$ values are preferred. 

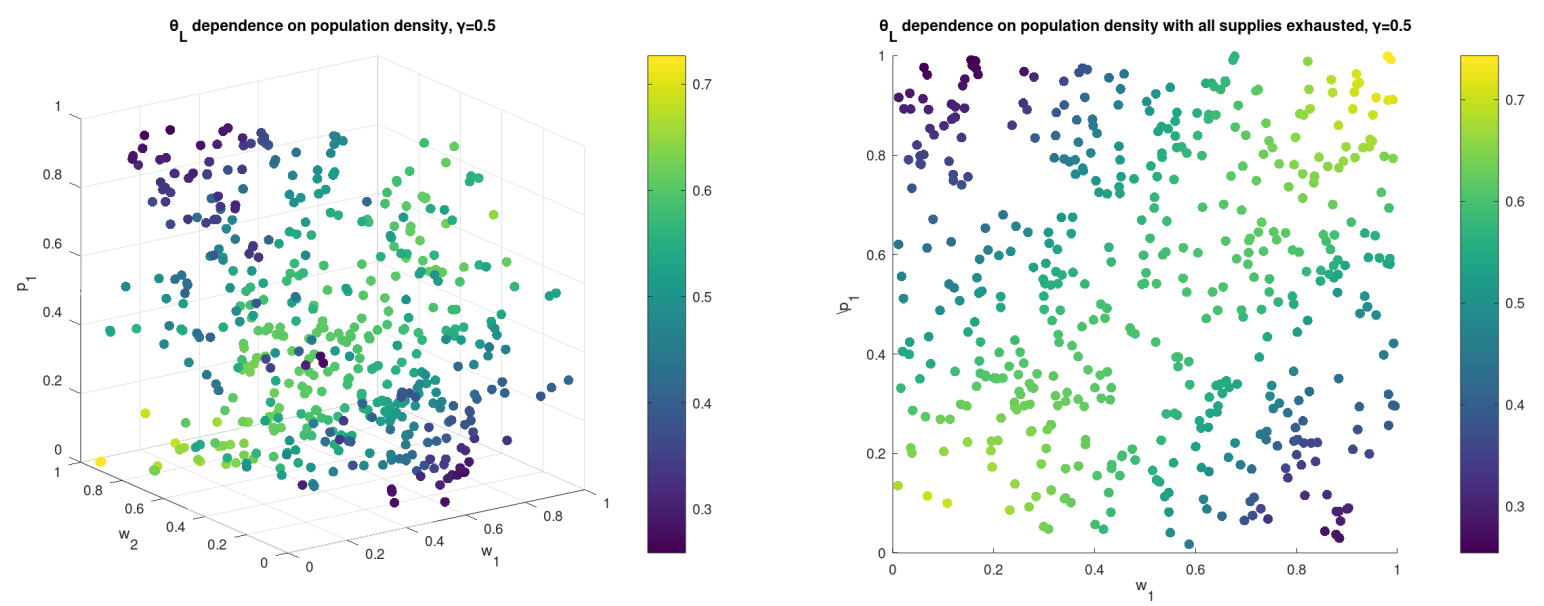

Figure 7. Two parameters are freely distributed: $\left(w_{1}\right.$ and $\left.p_{1}\right)$ while $\gamma=0.5, q_{1}=$ $\frac{p_{1}}{\sqrt{w_{1}}}-p_{1}$ and $q_{2}=\frac{1-p_{1}}{\sqrt{w_{2}}}-\left(1-p_{1}\right)$. In the left panel, $w_{2}$ is freely distributed with the constraint $w_{1}+w_{2} \leq 1$, whereas the right panel depicts the case where supplies are exhausted, with $w_{1}+w_{2}=1$.
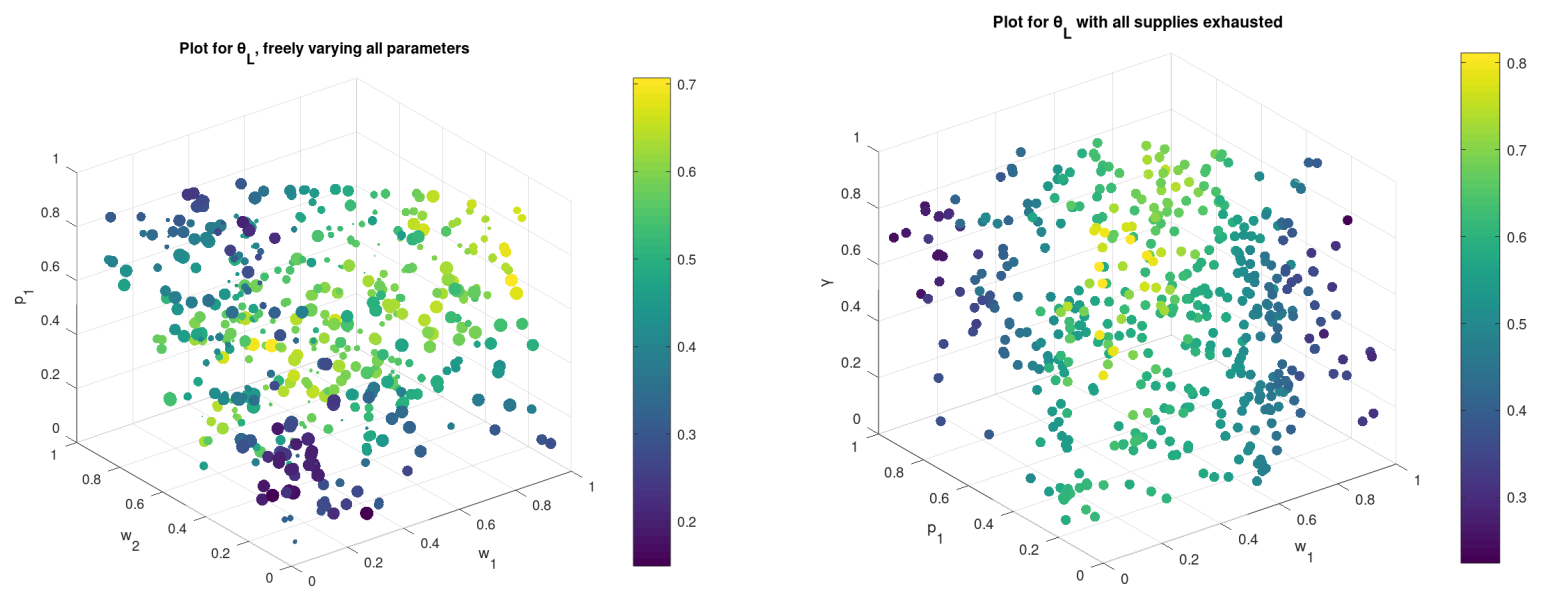

FiguRE 8 . Three parameters are freely distributed $\left(w_{1}, p_{1}, \gamma\right)$ with $p_{2}=1-p_{1}, q_{1}=$ $\min \left(\frac{p_{1}}{\sqrt{w_{1}}}-p_{1}, 1\right)$, and $q_{2}=\min \left(\frac{1-p_{1}}{\sqrt{w_{2}}}-\left(1-p_{1}\right), 1\right)$. In the left panel, $w_{2}$ is freely distributed with the constraint $w_{1}+w_{2} \leq 1$. The size of each point corresponds to the value of $\gamma$. The right panel shows the case where $w_{1}+w_{2}=1$.

\section{INTERPLAY OF SUPPLIES BETWEEN ZONES}

In this section, we further assume that the probability of running out of supplies depends in general on the supply strategies in both zones. We can write functions for $q_{1}$ and $q_{2}$ in terms of the parameters 
$x_{1}, x_{2}, w_{1}$, and $w_{2}$, noting $w_{1}+w_{2}=1$. Let

$$
q_{1}=\frac{x_{1}^{2}}{w_{1}}+\frac{x_{1} x_{2}}{2 w_{1} w_{2}}-\frac{x_{1}}{2 w_{1}} \text { and } q_{2}=\frac{x_{2}^{2}}{w_{2}^{2}}+\frac{2 x_{1} x_{2}}{5 w_{1} w_{2}}-\frac{x_{2}}{2 w_{2}}+\frac{1}{10}
$$

By inserting the above expressions for $q_{1}$ and $q_{2}$ into the followers' games and making the choice $\gamma=0.5$, the payoffs in each zone are:

$$
E_{1}=x_{1}\left(-\frac{x_{1}}{4}+\frac{3}{4}\right)+\left(1-x_{1}\right)\left(\frac{3 x_{1}}{4}+\left(\frac{x_{1}^{2}}{4 w_{1}^{2}}+\frac{x_{1} x_{2}}{8 w_{1} w_{2}}-\frac{x_{1}}{8 w_{1}}\right)\left(1-x_{1}\right)\right)
$$

and

$$
E_{2}=x_{2}\left(-\frac{3 x_{2}}{8}+\frac{5}{8}\right)+\left(1-x_{2}\right)\left(\frac{5 x_{2}}{8}+\left(-\frac{11}{25}+\frac{3 x_{2}^{2}}{5 w_{2}^{2}}+\frac{6 x_{1} x_{2}}{25 w_{1} w_{2}}-\frac{3 x_{2}}{10 w_{2}}\right)\left(1-x_{2}\right)\right) .
$$

The computations are a two-step process. First we distribute $w_{1} \in[0,1]$ with a step size of 0.05 and using the constraint $w_{1}+w_{2}=1$, then solve the followers' game for each pair of values. It is worth noting that different choices of $\gamma$ significantly modify the followers' payoffs. Using the equilibrium points $x_{1}^{*}$ and $x_{2}^{*}$ from these games, we can simultaneously solve for the values of $q_{1}$ and $q_{2}$ in the above formulae. Once $q_{1}$ and $q_{2}$ are known, we again solve the followers' game using those probabilities to gain $x_{1}$ and $x_{2}$. This finally allows us to optimize the leader's function to determine the optimal supply distribution between the two zones. The solutions to the game are given in Figure 9.
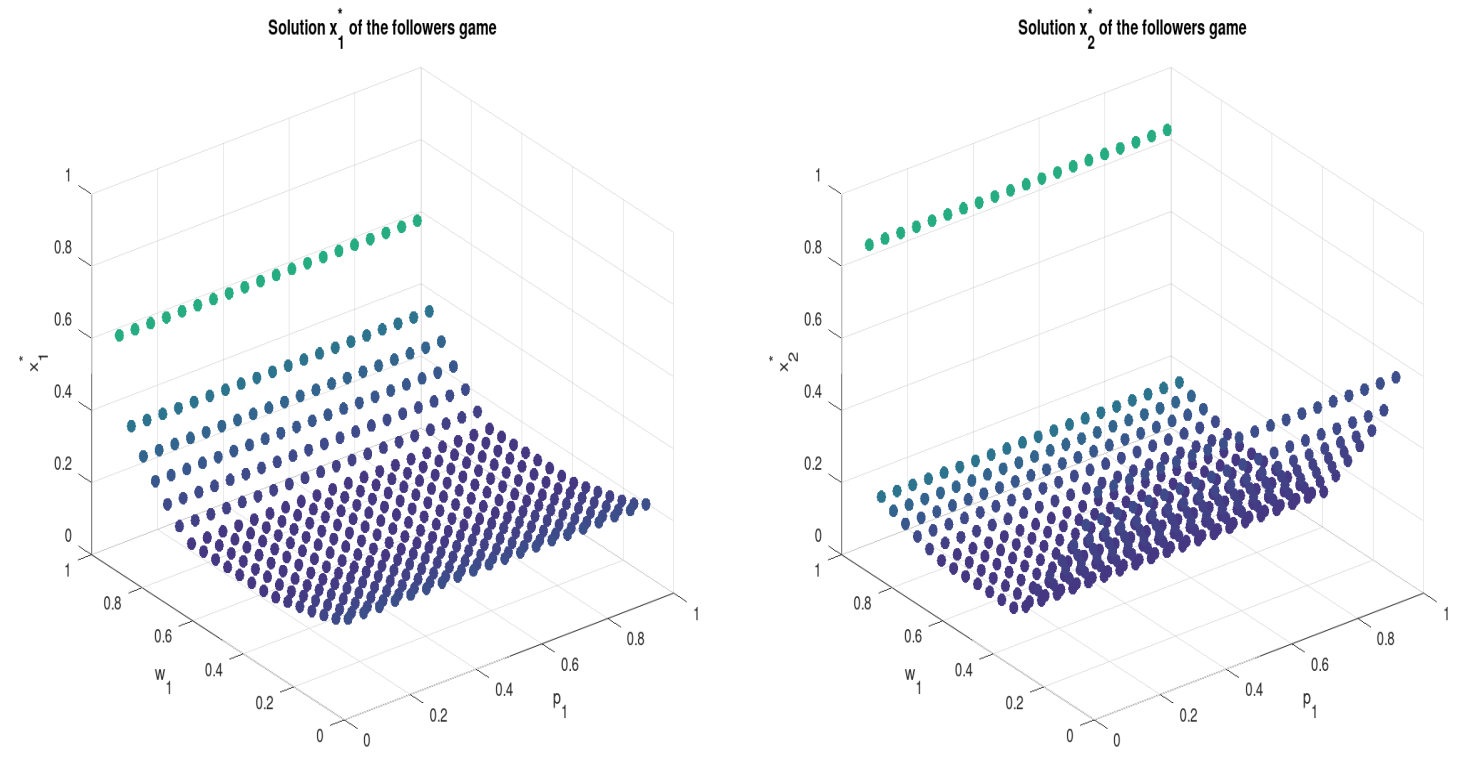

Figure 9. Solutions $x_{1}^{*}$ (left panel) and $x_{2}^{*}$ (right panel) of the game with $\gamma=0.5$, varying supply allocation and population between zones

To observe the effects of relative population sizes, we additionally freely distribute the parameter $p_{1}$, with the constraint $p_{1}+p_{2}=1$, before solving the followers' game. Similarly, to determine the impact of the incentive factor, we can vary $\gamma$ before solving the followers' game. This leads to the results in Figure 10 . 

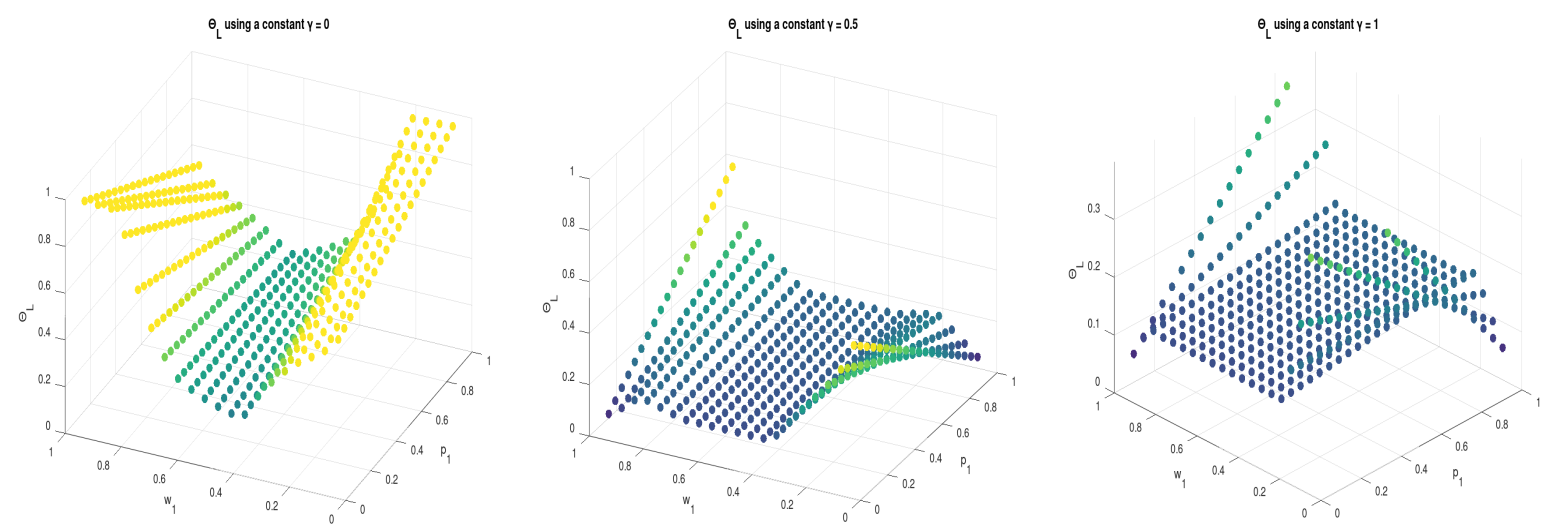

FIGURE 10. Leader's function with $\gamma \in\{0,0.5,1\}$, varying supply allocation and population between zones.

It turns out that $\gamma$ has a large effect on the leader's function, and in fact, the leader's values are optimal at the points where $\gamma=0$. This is in contrast to the previous models from sections 3 and 4 where high $\gamma$ values were preferred. This shows that with the interplay between both zones, larger objective function values are found if no incentive is provided. In examining population size, there is a strong correlation between the size of the population in a zone and the optimal weighting of supplies distributed to that zone. When one zone's population is double the other's or greater (i.e., when $p_{1} \geq 2 p_{2}$ ), there is a bias towards distributing most of the supplies to the larger zone. If both zones are of similar size, the leader has flexibility as to the weighting, with minimal impact on the objective function.

\section{DisCUSSION AND CONCLUSION}

This research examines the problem of resource allocation to multiple geographical zones to prepare for an adverse event using a bilevel game-theoretic approach. The focus is to incorporate the choices of the population on whether or not to prepare in advance, as well as zonal population densities and probabilities of running out of supplies. We use a leader-followers problem to optimise the supplies allocation to each zone for the leader, in order to supply as much of the population in advance as possible. The main factors impacting the leader's decision and allocation schemes are zonal population densities, incentivizing the acquisition of supplies in advance by population groups (for instance groups "at-risk"), and the probabilities of allocated supplies to be exhausted.

In the case of 2 zones with equal populations, the leader's function is optimal for values of $\gamma$ approaching 1, as in Figures 1, 2, and 3. This indicates that the leader should incentivize the purchase of supplies in order to maximize the objective function. With the additional assumption that available supplies depend on the population size of each zone, again, high $\gamma$ values are preferred. The functional form of the probability of running out of supplies is highly important: In the situation where supply availability is also dependent on the supply strategies in each zone, $\gamma=0$ is the best choice as in Figure 10. With the interdependence of both zones, neither zone should receive an incentive.

In the model from section 3, the result is straightforward that larger zones receive more supplies. As soon as the probability of running out of supplies takes a functional form, the optimal supply distribution is less clear, as is evident from Figure 8 In general, supplying the larger zone is still preferred. For the 
case in Section 5 with $q_{1}$ and $q_{2}$ based on the strategies of each player, if the zones are comparably sized, there is a large region in Figure 10 in which the objective function is roughly constant. This suggests that the leader has freedom in how to distribute supplies to the zones, without significantly affecting how much of the population supplies in advance. However, if the population sizes are dramatically different, then the best policy is to supply the largest zone.

Future research will focus on the case where the populations in the different zones are subject to interdependent constraints [4, 9].

\section{REFERENCES}

[1] N. Altay and W. G. Green, OR/MS research in disaster operations management, European Journal of Operational Research 175(2006), 475-493.

[2] N. Baker and L. G. Ludwig, Disaster preparedness as social control, Critical Policy Studies 12(2018), 24-43.

[3] M. Breton, G. Zaccour and M. Zahaf, A game-theoretic formulation of joint implementation of environmental projects, European Journal of Operational Research 168(2006), 221-239.

[4] M.-G. Cojocaru, E. Wild and A, Small, On describing the solution sets of generalized Nash games with shared constraints, Optimization and Engineering 19(2018), 845-870.

[5] G. Galindo and R. Batta, Review of recent developments in OR/MS research in disaster operations management, European Journal of Operational Research 230(2013), 201-211.

[6] T. Gossler, T. Wakolbinger, A. Nagurney and P. Daniele, How to increase the impact of disaster relief: A study of transportation rates, framework agreements and product distribution, European Journal of Operational Research 274(2019), 126-141.

[7] R. Lopes, Community disaster education, Proceedings of the Planning for an Earthquake in New Zealand Conference, IRL Conference Centre, Gracefield, Lower Hutt: Insitute of Geological and Nuclear Sciences, 2000.

[8] D. McLoughlin A Framework for Integrated Emergency Management, Public Administration Review 45(1985) (Special Issue: Emergency Management: A Challenge for Public Administration),165-172.

[9] T. Migot and M. G. Cojocaru, A parametrized variational inequality approach to track the solution set of a generalized nash equilibrium problem, European Journal of Operational Research 283(2020), 1136-1147.

[10] L. Muggy and J. L. Heier Stamm, Game theory applications in humanitarian operations: a review, Journal of Humanitarian Logistics and Supply Chain Management 4(2014), 4-23.

[11] A. Nagurney, P. Daniele, E. A. Flores and V. Caruso, A Variational Equilibrium Network Framework for Humanitarian Organizations in Disaster Relief: Effective Product Delivery Under Competition for Financial Funds, In: Kotsireas I., Nagurney A., Pardalos P. (eds) Dynamics of Disasters. DOD 2017. Springer Optimization and Its Applications, vol 140. Springer, Cham., pp. 109-133. https://doi.org/10.1007/978-3-319-97442-2/

[12] A. Nagurney and E. A. Flores A Generalized Nash Equilibrium network model for post-disaster humanitarian relief, Transportation Research Part E: Logistics and Transportation Review 95(2016), 1-18.

[13] NOAA National Centers for Environmental Information (NCEI) U.S. Billion-Dollar Weather and Climate Disasters (2019). https://www.ncdc.noaa.gov/billions/ accessed May 2020.

[14] D. Paton, Disaster preparedness: A social-cognitive perspective, Disaster Prevention and Management 12(2003), 210-216.

[15] K. J. Tierney, M. Lindell and R. Perry, Facing the Unexpected: Disaster Preparedness and Response in the United States, Joseph Henry Press, Washington DC, 2020. https://doi.org/10.17226/983

Corresponding author, Department of Mathematics and Statistics, University of Guelph, 50 Stone Rd E., Guelph, ON, N1G 2W1

E-mail address: mnahirni@uoguelph.ca

Department of Mathematics and Statistics, University of Guelph, 50 Stone Rd E., Guelph, ON, N1G 2W1 E-mail address: mcojocar@uoguelph.ca

Department of Mathematics and Statistics, University of Guelph, 50 Stone Rd E., Guelph, ON, N1G 2W1 Current address: Department of Mathematics and Industrial Engineering, Polytechnique Montral, Montral, Canada

E-mail address: tangi.migot@gmail.com 